\title{
A simple method for recording the path of a rat in an open field
}

\author{
O. GAPENNE, P. SIMON, and J. LANNOU \\ Laboratoire de Neurophysiologie Sensorielle, UFR des Sciences et Techniques de Rouen \\ Mont Saint Aignan, France
}

A method for recording the path of a rat during exploration or during a search task in an open field is presented. Its principle relies on the monitoring of the center of gravity of the rat. The open field lies on three strain gauges, on which output signals are sampled at a certain frequency by a digital acquisition system while the rat is walking on the platform. The discrete path is reconstructed off line, and several representative parameters are calculated. Some illustrative results are given.

Spatial orientation and memory have been intensively studied in laboratory animals mainly through the use of two experimental apparatuses: the radial maze (Olton \& Samuelson, 1976) and the water maze (Morris, 1981). In both procedures, animals are required to solve a spatial orientation task that involves the finding of a reward. Yet each of these experimental techniques has certain limitations. The radial maze drastically reduces the degrees of freedom of the animal's paths, and the water maze, which is very stressful, forces the animal (often a rat) to move in a rather artificial situation. The open field technique would therefore seem to suggest a better paradigm for studying spatial orientation, since it allows the animal to move in an environment that is more natural than water, and since the animal is free to walk in any direction during exploration or search. In fact, open fields have already been used in the study of some aspects of spatial orientation (Etienne, 1987; Muller, Kubie, \& Ranck, 1987; Poucet, Chapuis, Durup, \& Thinus-Blanck, 1986).

One problem with the open field is how to measure adequately the animal's performance in relation to the problem that the animal has to solve. One way to overcome this difficulty is to record the path of the animal and subsequently calculate all the parameters with which its motor and orienting behaviors can be characterized. This approach has been used, mainly with video tracking techniques (Eichenbaum, Kuperstein, Fagan, \& Nagode, 1987; Muller et al., 1987). Here, we will present an alternative method that allows one to record accurately the path of a rat moving freely in a classical open field and to calculate from this path significant parameters such as positions, linear and angular velocities, time moving and not moving, and so forth. This method, which can be used in many different situations for rats (we will give some

The authors wish to thank A. M. Bottreau, F. Lefebvre, B. Delahaye, and G. Lietout for their technical assistance. Requests for reprints should be sent to J. Lannou, Laboratoire de Neurophysiologie Sensorielle, UFR des Sciences et Techniques de Rouen, BP 118, 76134 Mont Saint Aignan cedex, France. examples), could easily be adapted for use with smaller or larger animals.

\section{METHOD}

The method consists of calculating the position of the center of gravity of the rat walking on the floor of a circular open field by computing three signals emitted by three strain gauge transducers localized in a triangular array at the periphery of the platform. The weight of the rat is divided into three components, each applied to one of the strain gauges, the magnitudes of which are inversely proportional to the distances between the center of gravity of the rat and the three transducers, respectively. The computation of the three analog signals, which gives the rat's position, is performed off line after the three signals have been sampled, digitized at a given frequency, and stored in a microcomputer. The rat's positions are given in $x$-, $y$-coordinates, with the origin at the center of the circular open field. The rat's successive spatial positions are separated by the same amount of time, depending on the sampling frequency, and it is easy to reconstruct the discrete path corresponding to the real one and to calculate the desired parameters.

Our open field is a circular platform, $75 \mathrm{~cm}$ in diameter, made of 3-mm-thick aluminum surrounded by a circular wall $30 \mathrm{~cm}$ high (these dimensions can be modified, provided that the rigidity of the platform is maintained). A door $(15 \times 15 \mathrm{~cm})$ that can be manually opened and closed allows the rat, coming from a startbox independent of the platform, to enter the open field.

In the floor of the open field, 16 holes arranged in a $4 \times 4$ matrix have been drilled. The centers of adjacent holes are $13 \mathrm{~cm}$ apart. In each of them, a small cylindrical aluminum cup (3 $\mathrm{cm}$ in diameter and $2.6 \mathrm{~cm}$ deep) is inserted. In these cups, rewards (food pellets or water) that the rat must find can be placed. An automatic system, which will be described in a subsequent section, has been designed to record the exploration of the cups by the rat. 


\section{Strain Gauge Transducer System}

The open field lies horizontally on three conventional strain gauges (Model Z8, Schenck S.A.) arranged in an equilateral triangle (Figure 1). The coupling between the platform and the active part of each transducer is ensured by a cone-shaped piece of aluminum whose apex is exactly $1 \mathrm{~cm}$ away from the edge of the open field. The strain gauges have been chosen to fulfill certain requirements: 1 . The total mass of the open field (platform + cups + rats + connections) must be less than three times the nominal charge of one transducer in order for the transducers to operate within their best working range (the nominal charge is the upper limit of the domain in which the errors are minimal). In our case, the total mass of the system was about $10 \mathrm{~kg}$, the nominal charge of the transducers being $5 \mathrm{~kg}$. 2 . The sensitivity of the strain gauges, which depends on their mechanical properties and on the power supply, must be sufficient to transduce accurately the displacements of a relatively light animal. The output signals from the strain gauges are small in amplitude, and they must be amplified to fit the input range of the $A / D$ converter. All the electronics (power supply, amplifiers, low-pass filters) will not be described here, since any electronic workshop can easily produce them.

\section{System for Recording Exploration of the Cups}

As stated earlier, the 16 cylindrical cups, which protrude below the floor of the platform, are arranged in a $4 \times 4$ matrix. In each cup, 4 diametrically opposed holes ( $5 \mathrm{~mm}$ in diameter, with their center $3 \mathrm{~mm}$ below the inferior surface of the platform) have been drilled and oriented so that they determine the lines and the columns of the matrix. At both extremities of each line and each column are fixed a small infrared emitter (distance range: $1 \mathrm{~m}$ ) and a photoelectric receptor, respectively (Baumer Electric). The emitters' infrared beams (which do not need a particular setting, since they are sufficiently focused) of the lines cross those of the columns exactly at the center of each cup, and so, when the rat puts its nose into a cup to explore it or to catch the reward, it interrupts two beams, one of a line and the other of a column, thus activating the corresponding receptors. The activated receptors trigger specific DC potentials, which have been chosen so that the sum of the DC potential of one given line receptor plus the DC potential of one given column receptor is unique and coded for the cup situated at the crossing of that line and that column. The 16 cups are thus represented by 16 different DC potentials ranging from 0.3 to $4.8 \mathrm{~V}$ sampled together with the three output

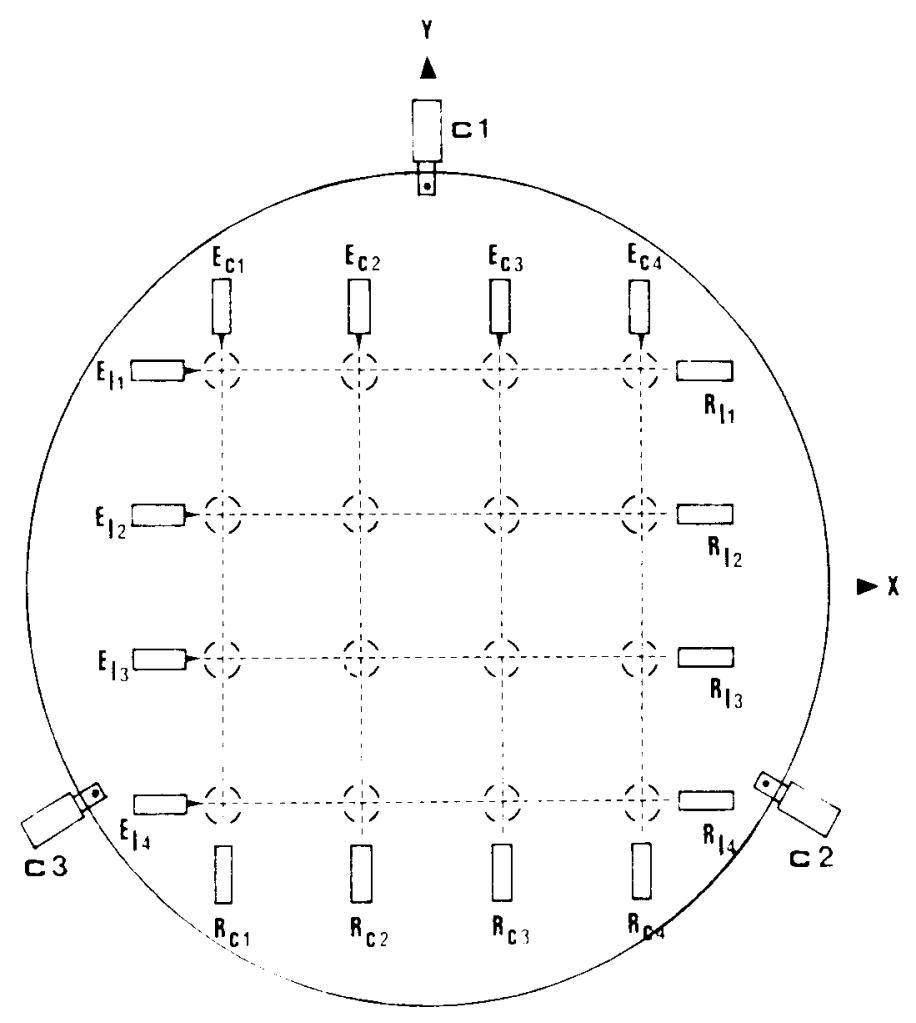

Figure 1. Schematic drawing of the open field. Ec1, Ec2, .., and El1, E12, $\ldots$ are infrared emitters of columns and lines, respectively. Rc1, Rc2, ..., and $R 11, R 12, \ldots$ are receptors of columns and lines, respectively. $\mathrm{C1}, \mathrm{C2}$, and $\mathrm{C} 3$ are the three strain gauges. 
signals from the strain gauges. Although it is not technically possible, with this system, to distinguish between head-in-cup behavior and interruption of the beams by some other body part, visual observation of many rats has shown that the latter almost never occurs.

\section{Data Acquisition}

Data are acquired through an $\mathrm{A} / \mathrm{D}$ converter board plugged into an IBM PC-compatible microcomputer. The A/D converter is a 12-bit, $0-5-\mathrm{V}, 16$-channel converter (unipolar inputs) with an acquisition time of $100 \mu \mathrm{sec}$ per channel. The acquisition program allows one to select the number of channels, the frequency of sampling, and the maximal duration of one acquisition, with interruption when desired by striking a key on the keyboard. In our tests, we have chosen the following parameters: 4 channels ( 3 for the strain gauges and 1 for the cups exploration); maximal duration of $360 \mathrm{sec}$; and a sampling frequency of 4 or $6.67 \mathrm{~Hz}$.

The maximal file size was then 360 (seconds) $\times 4$ (channels) $\times 6.67$ (frequency) $\times 2$ ( 2 bytes for one conversion) $=19,210$ bytes. These settings gave a satisfactory resolution and relatively small files that could be handled and processed easily in a short time.

\section{Data Processing}

The data processing program has been written in Turbo Pascal (Borland Intl). It is composed of several procedures that calculate the desired parameters.

First, an intermediate file with only the three signals from the strain gauges is created to facilitate the filtering (moving averaging); then, the $x-, y$-coordinates are calculated in, using the following equations:

$$
\begin{aligned}
& x=\frac{S_{3}-S_{2}}{S_{1}+S_{2}+S_{3}} \times K_{x} \\
& y=\frac{2 S_{1}-S_{2}-S_{3}}{S_{1}+S_{2}+S_{3}} \times K_{y}
\end{aligned}
$$

$S_{1}, S_{2}$, and $S_{3}$ are the digitized signals of the three strain gauges $(\mathrm{C} 1, \mathrm{C} 2$, and $\mathrm{C} 3$, respectively; see Figure 1) sampled at the same time. As already stated, the signals are not sampled exactly at the same time but with delays of $100 \mu \mathrm{sec}$. This sequential sampling introduces a negligible error, since the rat cannot travel more than a tenth of a millimeter in $300 \mu \mathrm{sec} . K_{x}$ and $K_{y}$ are scaling constants that depend on the sensitivity and the gain of the strain gauges system and must be estimated, prior to any utilization of the apparatus, by calibration tests: Small masses (ranging from 100 to $300 \mathrm{~g}$ ) were placed for $20 \mathrm{sec}$ at known $x$-, $y$-coordinates, the corresponding signals from the three transducers were sampled and averaged, $K_{x}$ and $K_{y}$ were calculated from Equations 1 and 2 for each mass and each position, and, finally, mean values for $K_{x}$ and $K_{y}$ were computed. The measurement errors correspond to the difference between the actual $x$-, $y$ - coordinates of a mass and the ones calculated by using these mean values of $K_{x}$ and $K_{y}$. Figure 2 shows these measurement errors along the $x$ and $y$ axes in different sectors of the open field for a $250 \mathrm{-g}$ mass. They are maximal at the periphery of the open field but never exceed $1.2 \mathrm{~cm}$, the mean absolute errors being 0.3 and $0.44 \mathrm{~cm}$ along the $x$ and $y$ axes, respectively.

We have also tested the system in dynamic conditions. To do this, we compared the parameters characterizing the actual movement of a $250-\mathrm{g}$ wheel turning around the open field along a circle $62 \mathrm{~cm}$ in diameter (constant angular velocity, $57.6^{\circ} / \mathrm{sec}$; linear velocity, $31.1 \mathrm{~cm} / \mathrm{sec}$ ) to the ones calculated by the program after sampling of this movement at $6.67 \mathrm{~Hz}$. The parameters returned by the program were: angular velocity, $57.6 \% \mathrm{sec}$; diameter of the circles, $60.9 \mathrm{~cm}$; linear velocity, $30.6 \mathrm{~cm} / \mathrm{sec}$. The latter value exceeds largely the maximal rat's velocity that we have observed in our tests, since we have rarely found animals running faster than $20-22 \mathrm{~cm} / \mathrm{sec}$. All these preliminary tests were necessary to determine the accuracy of the technique. In addition, they have shown that after a warm-up period of $30 \mathrm{~min}$ there is no drift in the strain gauge signals and that the system is perfectly reliable for long periods of time $(8 \mathrm{~h}$ per day for 6 consecutive months).

The successive $x$-, $y$-coordinates are saved in a file, and they are used for the display of the rat's path on the screen and printer and for the calculation of several quantitative parameters that characterize the spatiotemporal organization of the rat's behavior:

1. Duration of acquisition-either the maximal time allowed or the time the rat took to find the reward. The time the rat is moving and the time the rat is not moving are also calculated. The rat is considered as not moving when two successive positions are separated by a distance inferior to a threshold fixed by the program (in our case, $0.25 \mathrm{~cm}$ ); this, of course, can be modified.

2. Length of path-the total distance the rat travels on the platform during its exploration or the search task.

3. Mean linear velocity-the path length divided by the time the rat was moving.

4. Mean angular velocity-gives information about the tendency of the rat to turn symmetrically or not toward the left and right. By discrete sampling, a curvilinear path of the rat is transformed into a sequence of rectilinear segments. The angles between each pair of adjacent segments are calculated, summed (angles to the left and right are positive and negative, respectively), and divided by the elapsed time to obtain the mean angular velocity. A histogram of the distribution of angles is displayed in Figure 3.

5. Sinuosity-This parameter, defined by Bovet (1981, 1985), gives an estimation of the spatial complexity of the animal's path (i.e., whether this path is more or less straight); it is independent of the sampling frequency and is calculated from the following equation (for further explanations, see Benhamou, 1986; Clement, Fouillet, \& Mimouni, 1987): 


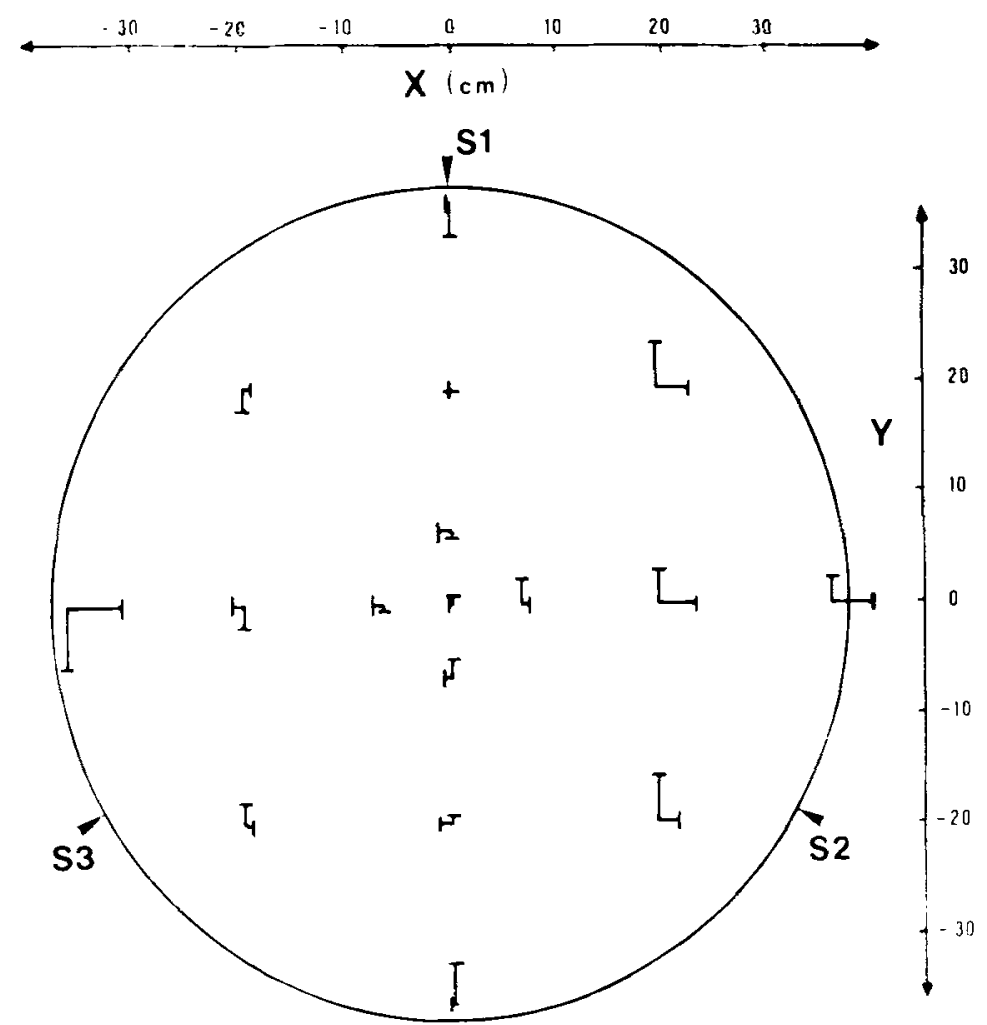

Figure 2. Measurement errors along the $x$ - and $y$-axes in different sectors of the open field. These errors are the differences between the real coordinates of a 200-g mass and the corresponding ones calculated by the program; for precision, they are drawn at a scale 10 times larger than the one (shown by the $x$ - and $y$-coordinates) used for the open field.

$$
S=\frac{\sigma_{a}}{\sqrt{P_{m}}}
$$

where $S$ is the sinuosity, and $\sigma_{a}$ is the standard error of the angular velocity (determined when the mean angular velocity is calculated). $P_{m}$, the mean step (i.e., the mean length of all the segments joining the couples of successive positions of the rat), is determined when the length of the path is calculated by dividing this length by the number of steps.

6. The propensity of the rat to stay in specific areas of the open field-The program divides the platform surface into three virtual concentric zones of equivalent areas, called external, medial, and central zones. The cumulated numbers of positions of the rat in each zone are calculated and normalized.

7. Exploration of the cups-The procedure for processing the exploration of the cups gives the total numbers of cups the rat has explored during a trial; the number of times each cup has been explored, and for how long; and the sequence in which the different cups have been explored.

All these data enable the comparison between control and experimental rats and allow one to measure the mo- tor (distance traveled, linear and angular velocities, sinuosity), motivational (time moving and not moving), and attentional (space occupation, exploration of the cups) aspects of their behavior.

\section{RESULTS}

It is not our purpose in this paper to detail the results obtained with our apparatus. Some examples (Figure 3) are given simply to demonstrate the discriminative power of the method in comparing the orienting behavior of rats learning different tasks and in comparing control and lesioned rats (in this case unilabyrinthectomized animals) placed in the same situation.

The path in Figure $3 \mathrm{~A}$ is that of a rat exploring the open field for the first time. It is long $(19.9 \mathrm{~m}$ in $360 \mathrm{sec})$ and almost entirely confined to the periphery of the platform (thigmotactism); this qualitative observation is confirmed by the relative "occupation" of the three zones (79\%, $15 \%$, and $6 \%$, in the external, medial, and central zones, respectively). The mean linear velocity is low $(8.2 \mathrm{~cm} / \mathrm{sec})$, and the mean angular velocity $(-10.3 \% / \mathrm{sec})$ indicates that the rat did turn mainly rightward. The sinuosity is relatively high $\left(0.8 \mathrm{rad} \times \mathrm{cm}^{-1 / 2}\right)$, and the rat did not find the food pellet (marked as a star in one cup). After 14 

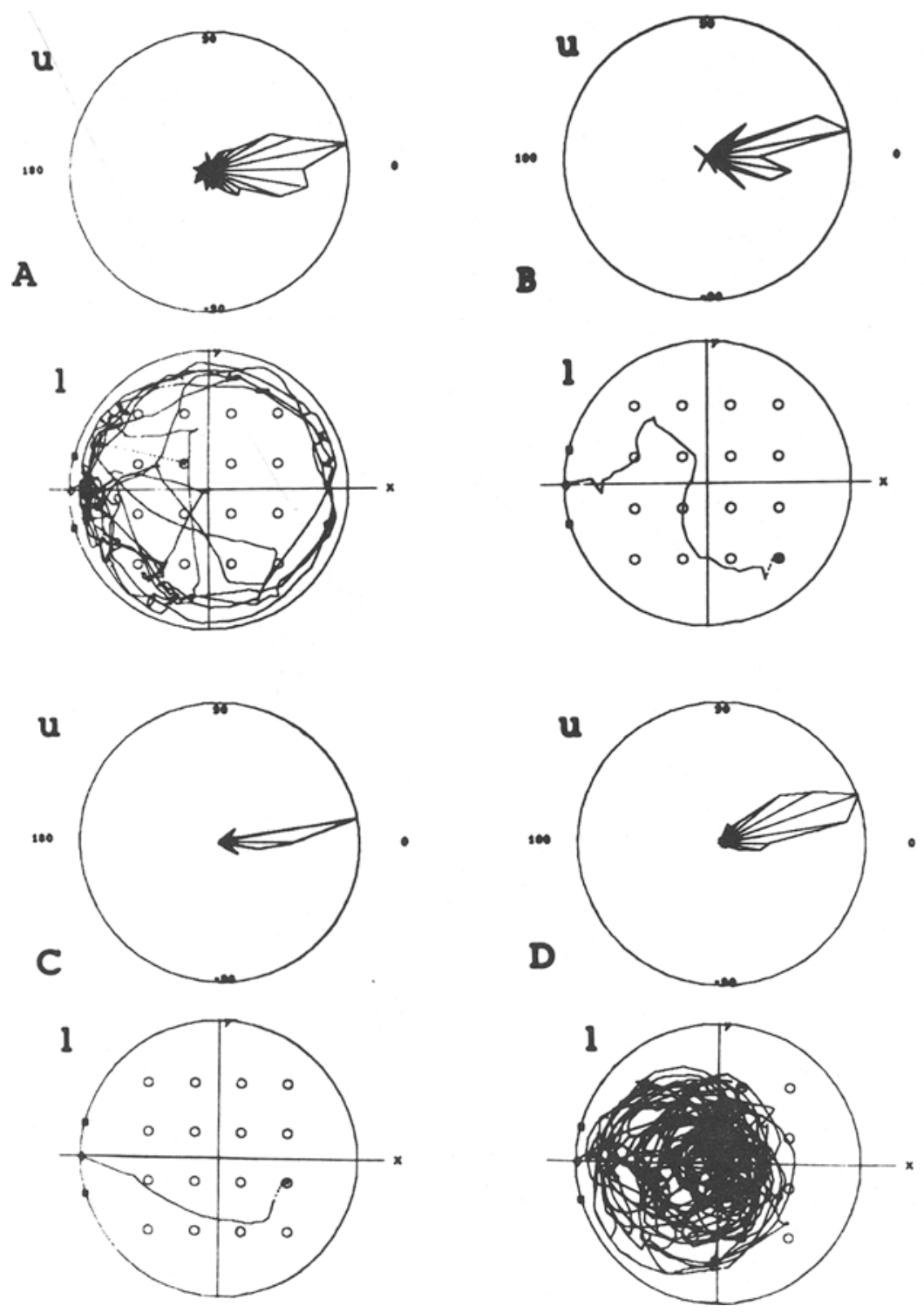

Figure 3. Some examples of paths recorded with rats in different conditions. For each condition, the lower diagram ( $L$ ) represents the path of the rat in the open field; the star shows the cup containing the food pellet, and the dashed line joins the last position of the rat at the end of the trial to that cup. The upper diagram $(\mathrm{U})$ is the distribution of all the angles present in the same path, displayed as a circular histogram of 36 classes: $(+5,+15),(+15,+25), \ldots(+175,-175),(-175,-165), \ldots(-5,+5)$. All the classes have their origin at the center of the circle; they are normalized relative to the modal class whose frequency is referred to as 1 and corresponds to the radius of the circle.

trials, the pattern of displacement of the same rat is very different (Figure 3B); the path is short $(0.98 \mathrm{~m})$, and the rat finds the reward quickly $(11.5 \mathrm{sec}$; linear velocity, $9.34 \mathrm{~cm} / \mathrm{sec})$. The sinuosity is lower $\left(0.42 \mathrm{rad} \times \mathrm{cm}^{-1 / 2}\right)$ than in the first trial.

The path shown in Figure $3 \mathrm{C}$ is still different; it is short $(0.53 \mathrm{~m}$ ) and nearly straight (sinuosity, $0.13 \mathrm{rad} \times \mathrm{cm}-1 / 2)$, since the rat went directly to the cup containing the food reward. This path is that of a rat trained for 30 trials to find a food pellet that remained in the same cup, whereas the rat of Figure 3B was trained to find a reward that was pseudorandomly distributed into one of the 16 cups so that it was never in the same cup in successive trials.

Figure 3D exhibits the path of an untrained rat that had been unilaterally labyrinthectomized (left side) $24 \mathrm{~h}$ prior to the test. The path is extremely long $(44.8 \mathrm{~m})$, and there is no thigmotactism $(13 \%, 33 \%$, and $54 \%$ in the external, medial, and central zones, respectively); the linear velocity is high $(15.3 \mathrm{~cm} / \mathrm{sec})$, and the angular velocity $(73.6 \% \mathrm{sec})$ clearly shows the tendency of the rat to turn 
toward the lesioned side. This propensity is also shown by the distribution of the angles shifted toward positive values.

\section{CONCLUSION}

The technique we have developed allows one to record the paths of rats moving freely on a platform and to calculate parameters representative of these paths. This technique is accurate; it can be adapted to many experimental paradigms; it needs absolutely no preparation of the animals; it can be used in complete darkness and allows one to control the visual environment entirely; it is not expensive; the hardware and software are easy to develop; and it represents an alternative to more expensive video tracking techniques.

\section{PROGRAM AVAILABILITY}

A copy of the software may be obtained by sending a formatted 5.25-in. diskette to the authors.

\section{REFERENCES}

Bovet. P. (1981). Modèles aléatoires de l'exploration. Bulletin Intérieur de la SFECA, 1, 11-21.

BOVET, P. (1985). Les déplacements au hasard chez les êtres vivants.
Unpublished doctoral dissertation, University of Aix-Marseille II, France.

Benhamou, S. (1986). Déplacement des mammifères dans leur domaine vital: Essai de modélisation. Unpublished doctoral dissertation, University of Aix-Marseille II, France.

Clement, P., Fouillet, P.. \& Mimouni, M. (1987). Clinocinèse. orthocinèse ou choix d'indices pour analyser des déplacements non orientés obtenus en trajectométrie automatique. Bulletin Intérieur de la SFECA, 2, 139-146.

Eichenbaum, H., Kuperstein, M., Fagan, A., \& Nagode, J. (1987). Cue-sampling and goal-approach correlates of hippocampal unit activity in rats performing an odor-discrimination task. Joumal of Neuroscience, 7, 716-732.

EtienNe, A. S. (1987). The control of short-distance homing in the golden hamster. In P. Ellen \& C. Thinus-Blanc (Eds.), Cognitive processes and spatial orientation in animals and man (NATO ASI Series, pp. 233-251). Dordrecht: Martinus Nijhoff.

Morris, R. G. M. (1981). Spatial localization does not require the presence of local cues. Learning \& Motivation, 12, 239-260.

Muller, R. U., Kubie, J. L., \& Ranck, J. B. (1987). Spatial firing patterns of hippocampal complex-spike cells in a fixed environment. Journal of Neuroscience, 7, 1935-1950.

Olton, S. D., \& SAmuelson, R. J. (1976). Remembrance of places passed: Spatial memory in rats. Journal of Experimental Psychology: Animal Behavior Processes, 2, 97-116.

Poucet, B., Chapuis. N., Durup, M., \& Thinus-Blanc, C. (1986). A study of exploratory behavior as an index of spatial knowledge in hamsters. Animal Learning \& Behavior, 14, 93-100.

(Manuscript received February 16, 1990; revision accepted for publication June 20. 1990.) 